\title{
DO PATIENTS REPORT QUALITY OF LIFE IMPROVEMENTS AFTER FITTING OF THEIR UNILATERAL BONE ANCHORED HEARING DEVICE?
}

MEGHJI, S., MILLINGTON, A., COLLETT, A. \& HANIF, J.

\section{Introduction:}

Changes in patient's quality of life must be considered when looking at the overall success of a surgical intervention. Patient reported outcome measures (PROMS) are widely used in surgery to try and assess the qualitative impact of a particular intervention or device. The Glasgow Benefit Inventory (GBI) is a validated questionnaire used fairly frequently in otolaryngology patients looking at generic PROMS. [1] It was initially designed to be used post-intervention to assess the impact of the procedure. The Glasgow Health Status Inventory (GHSI) is similar to the GBI questionnaire but it can be used pre-intervention and postintervention. Both the $\mathrm{GBI}$ and $\mathrm{GHSI}$ contain 18 questions and take about 10 minutes to fill in. Responses are scaled and averaged. The poorest outcome can score -100 and the best outcome can score +100 . In 1977 Tjellstrom et al. ${ }^{[2]}$ were the first clinicians to implant a bone anchored hearing aid (BAHA). 40 years later, more than 150,000 patient have regained their hearing with a BAHA. The audiological benefits of bone anchored hearing devices have been well document in many previous studies. ${ }^{[3-4]}$ Many of these quality of life studies were performed with older devices and different surgical techniques. This study assesses the quality of life improvement when a bone anchored hearing device is used.

\section{Methodology:}

Prospective cross sectional study of all adult patients who underwent a unilateral BAHA insertion in a large teaching hospital in the East of England over four years. Patients completed a preoperative GHSI questionnaire about their quality of life before their BAHA. They then completed a post-operative GHSI questionnaire three months after the BAHA was loaded onto the implant. Both questionnaires were completed by the audiologist. Patients were included in the study if they were older than 18 years old, had a unilateral BAHA and a completed pre-operative GHSI.

\section{Results:}

121 patients were approved and listed for a BAHA between March 2012 to March 2017. Many patients were excluded: 33 were still awaiting their surgery, 23 have recently had surgery were awaiting the post-operative questionnaire. The other excluded cases: 11 were having a second BAHA placed, 5 patients did not have pre-operative scores noted, 3 abutments fell out and 1 minimal access procedure failed.

45 patients had a unilateral BAHA placed and completed both questionnaires. There were 19 females and 26 males.

\section{Reason for unilateral BAHA}

Using the GHSI questionnaire the total score went from -23 preoperatively to +55 post-operatively. The general benefit score went from -39 to +69 . The physical score went from -25 to +31 . The social score went from +36 to +14 . Patients scores were not normally distributed so a non-parametric Wilcoxon Signed-Rank test was performed to compare the mean scores pre-BAHA GHSI and the post-BAHA GHSI. The results from the general score (Z-value $=-5.8413)$ showed a statistically significant improvement from the pre-BAHA to the postBAHA score $(p<0.01)$. The results from the total GHSI score $(Z$-value $=$ -5.7301) also showed a statistically significant improvement from the pre-BAHA to the post-BAHA score $(p<0.01)$

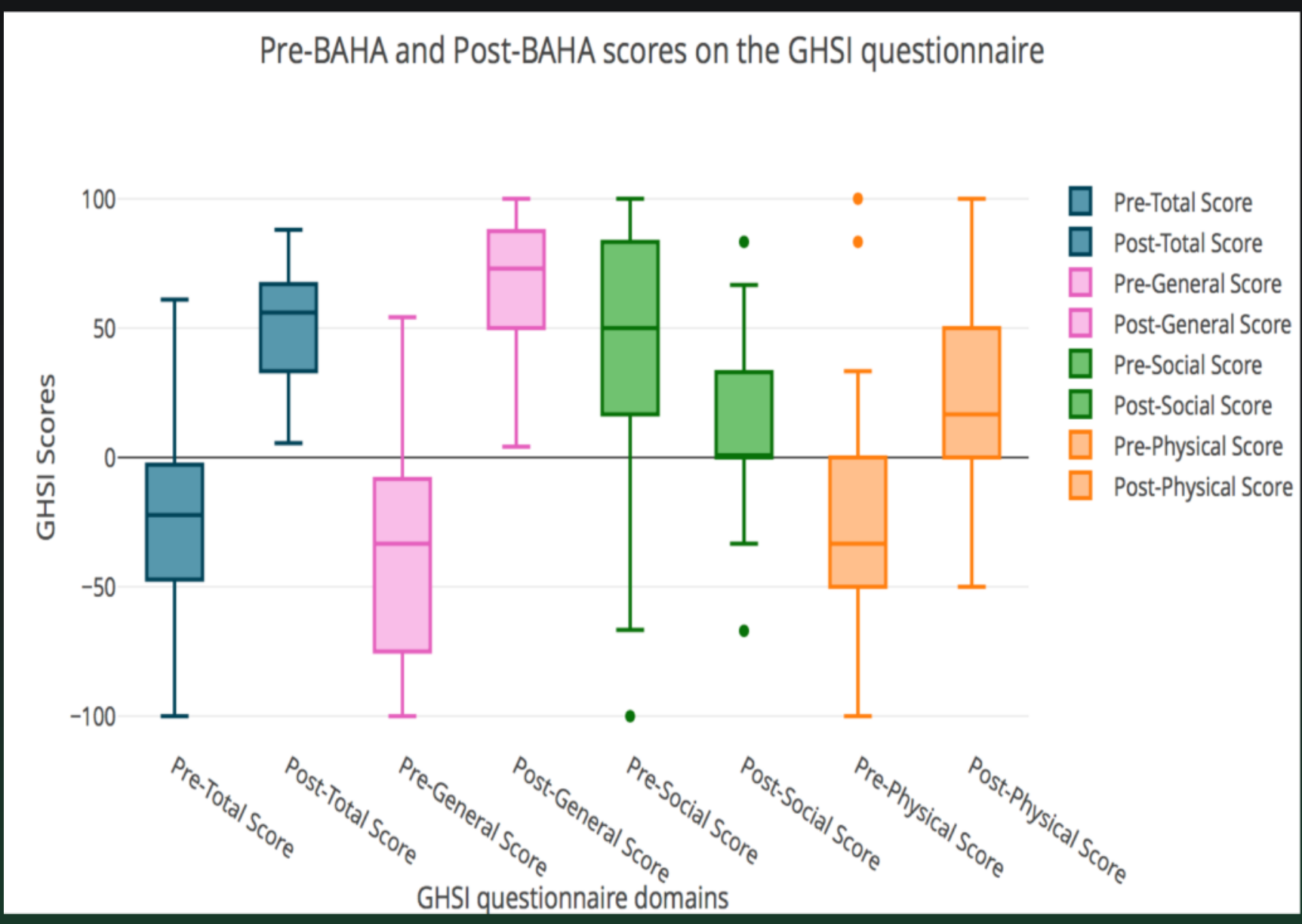

Discussion:

This study clearly shows a vast improvement in patient's perceived quality of life after BAHA surgery. The post-operative total and general scores on the GHSI showed a statistically significant improvement when compared to the pre-operative scores. The innovative advances in devices, reduction in soft tissue trauma during surgery along with a dedicated team dealing with BAHA patients has led to streamlining the patient's pathway and increasing their quality of life. Additionally, the study introduces the novel use of the GHSI before the surgical intervention and after the intervention to get a matched comparison. The GHSI questionnaire is easy to complete however it does have its limitations. Using the GHSI pre and post intervention can result in misleading interpretation of the raw score. In our cohort, patients reported very good social support prior to the intervention scoring +36 on the social scale. Post-operatively patients did not report any changes to their social support therefore scoring +14 on the social scale. The decline in score may lead to misinterpretation that the procedure had a negative impact on the patient's quality of life.

\section{Conclusion:}

In conclusion, this study adds to the body of evidence that suggests that patients fitted with a unilateral BAHA report quality of life improvements.

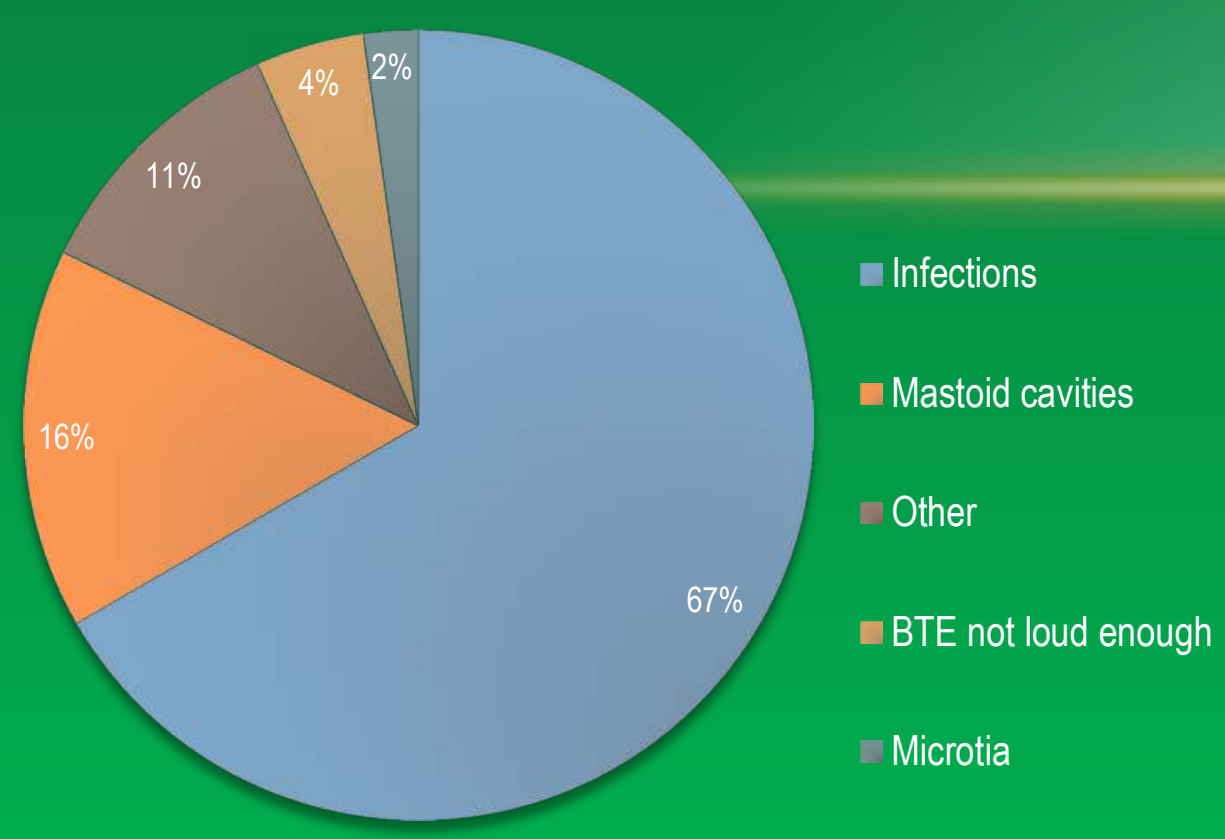

